\title{
BMJ Reporting of lifetime fractures: Open methodological considerations and results from the Thai Cohort Study
}

\author{
Janneke Berecki-Gisolf, ${ }^{1}$ Rod McClure, ${ }^{1}$ Sam-ang Seubsman, ${ }^{2}$ Adrian Sleigh, ${ }^{3}$ \\ Thai Cohort Study team ${ }^{i}$
}

To cite: Berecki-Gisolf $\mathrm{J}$, McClure R, Seubsman S-ang, et al. Reporting of lifetime fractures: methodological considerations and results from the Thai Cohort Study. BMJ Open 2012;2:e001000. doi:10.1136/bmjopen-2012001000

- Prepublication history and additional material for this paper are available online. To view these files please visit the journal online (http://dx.doi.org/10.1136/ bmjopen-2012-001000).

For author footnote see end of the article.

Received 10 February 2012 Accepted 24 July 2012

This final article is available for use under the terms of the Creative Commons Attribution Non-Commercial 2.0 Licence; see http://bmjopen.bmj.com

For numbered affiliations see end of article

\section{Correspondence to} Dr Janneke Berecki-Gisolf; janneke.berecki-gisolf@ monash.edu

\section{ABSTRACT}

Objectives: To provide estimates of fracture incidence among young adults in Thailand.

Design: Cross-sectional analysis of a large national cohort.

Setting: Thailand.

Participants: A total of 60569 study participants residing nationwide responded to the 2009 follow-up survey; $55 \%$ were women and median age was 34 years (range 19-92).

Outcome measures: Self-reported lifetime fractures, along with age at fracture. Fracture incidence rates per person-year were then compared using lifetime fracture reports, and again selecting only fractures reported for the last year. Incidence rates were compared by age and sex.

Results: 18010 lifetime fractures were reported; $11645(65 \%)$ by men. Lifetime fracture prevalence was $30 \%$ for men and $15 \%$ for women. Lifetime incidence per 10000 person-years was 83; analysing only fractures from the last year yielded a corresponding incidence rate of 187 . For ages 21-30, fractures per 10000 person-years were more common among men than women (283 (95\% Cl 244 to 326) and 150 (130 to 173 ), respectively); with increasing age, rates decreased among men and increased among women (for ages 51-60, 97 (58 to 151) and 286 (189 to 417), respectively).

Conclusions: Large-scale surveys provide a feasible method for establishing relative fracture incidence among informative subgroups in a population. Limiting analyses to fractures reported to have occurred recently minimises bias due to poor recall. The pattern of selfreported fracture incidence among Thais aged 20-60 was similar to that reported for Western countries: high falling rates in young men and high rising rates in older women.

\section{INTRODUCTION}

Fractures are an important public health burden. Descriptive epidemiological data on the incidence and distribution of fractures in the population are relevant for the provision of health services, identifying trends and

\section{ARTICLE SUMMARY}

Article focus

- In developing and newly industrialised countries, epidemiological data on fractures usually cannot be derived from large administrative datasets; we have therefore developed other means to study the occurrence of fractures.

- We illustrate and recommend a robust method limiting the effects of recall bias and estimating fracture incidence using direct population surveys.

- An additional aim of the study is to provide relative fracture incidence among young Thai adults using a 2009 4-year follow-up survey of a large national TCS.

\section{Key messages}

- Limiting analyses to fractures reported to have occurred recently minimises bias due to poor recall. Survey of an educated subpopulation provided a feasible alternative for establishing relative fracture incidence.

- The relative self-reported fracture incidence among adult Thai men and women between 20 and 60 years was similar to that reported for Western countries such as the USA and the UK.

Strengths and limitations of this study

- The strengths of the study are the very large sample size, the detailed questions on fractures and the careful consideration of recall bias in the analysis.

- The accuracy of self-report is assisted by education.

- The gold standards concerning fractures (x-rays and medical records) were not available for our large study population.

informing preventative strategies. Population fracture distributions have been described previously using administrative data, including x-ray reports, ${ }^{1-4}$ and using survey data, ${ }^{5}$ with widely varying results. Administrative datasets of patients attending outpatient fracture clinics have the advantage of accurate fracture ascertainment, but do not capture patients admitted to hospital, patients treated in primary care or fractures that do not result in 
medical intervention. ${ }^{5}$ Furthermore, to determine actual fracture incidence based on (administrative) hospital data, an estimate of the hospital catchment population is required, which is usually not feasible particularly for large surgical centres. Survey data capture all fractures regardless of medical intervention, provided there is accurate recall of the event by the study participants. Self-report of life events, however, is prone to telescoping (inaccurately reporting distant events as having occurred more recently) and fall-off (events reported in previous surveys may, in subsequent surveys, not be reported as having ever occurred). ${ }^{6}$

In developing countries and newly industrialised countries, large administrative datasets of routinely collected medical information may not be readily available and estimates of fracture occurrence must rely on other means. Recognising the importance of survey data in establishing fracture incidence in developing and newly industrialised countries, we present a method for quantifying and resolving the effect of poor recall on self-reported lifetime fracture incidence. In this study we also provide estimates of relative fracture incidence among young and middle-aged adult men and women in Thailand, using the 2009 4-year follow-up survey of a large national Thai Cohort Study (TCS).

\section{METHODS}

Study population and data collection

The data derived from the 2009 follow-up survey of the TCS, which is an ongoing community-based study of adult distance learning Sukhothai Thammathirat Open University (STOU) students residing throughout the country. In 2005, the STOU student register listed about 200000 names and addresses: a baseline 20-page questionnaire was sent to each student and 87134 (44\%) replied. The baseline characteristics of cohort participants $^{7}$ and comparisons with the adult population of Thailand ${ }^{8} 9$ have been reported previously: the STOU cohort has a slightly higher proportion of females than the general Thai population $(54.7 \%$ vs $50.5 \%)$; more young adults $(51.5 \%$ vs $23.9 \%$ were aged between 21 and 30 years) and fewer people aged over $50(2 \%$ vs $24.7 \%) ;{ }^{8}$ the age distribution of the STOU cohort is shown in figure 1. Study participants were also less likely to be married and more likely to have completed junior high school; geographically the main regions in Thailand are well represented in the STOU cohort. ${ }^{8}$

Overall the cohort represents well the geo-demographic, ethnic, occupational and socioeconomic status of the young-adult Thai population. This is because most Open University students are unable to leave their locations to attend an on-campus university fulltime: most already have established jobs and family responsibilities, and are of modest economic circumstances. However, they are better educated than the general Thai population and thus are able to respond to complex health questionnaires. In 2009, a follow-up survey was sent and 60569 (>70\%) participants replied: 55\% were women and the median age was 34 years (range 19-92). Data scanning, verifying and correcting were conducted using Scandevet, a programme developed by a research team from Khon Kaen University. Further data editing was completed using SQL and SPSS software.

\section{Ethical considerations}

Ethics approval was obtained from Sukhothai Thammathirat Open University Research and Development Institute (protocol 0522/10) and the Australian National University Human Research Ethics Committee (protocols 2004344 and 2009570). Informed written consent was obtained from all participants.

\section{Measures}

The core question asked was 'In your life have you ever experienced a fracture to the areas of your body mentioned below? If so please place a cross in the Yes box and indicate the age at which the fracture occurred' followed by a list: finger/toe; wrist; arm; collarbone; rib; skull; face/jaw/nose; neck; back; pelvis; leg; ankle; and other. Skull fractures may have been over-reported (by being confused with skull trauma due to the wording used in the original Thai questionnaire) and as this is the subject of further investigation skull fractures are not
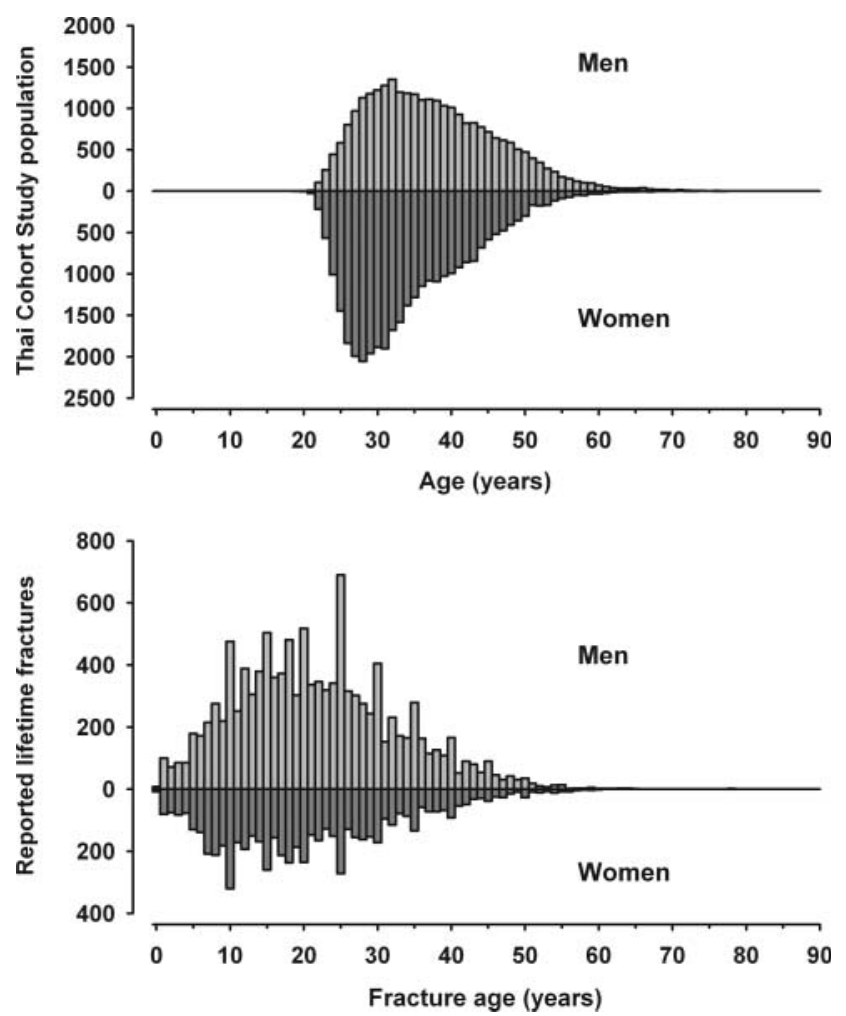

Figure 1 Top: histogram of Thai Cohort Study (TCS) participant age. Bottom: histogram of the total lifetime fractures reported by TCS participants: over-reporting of fractures can be seen at ages such as 10,15, 18, 25, 30, 35 and 40 . 
Table 1 Lifetime prevalence of fractures in Thai cohort members by age and sex

Lifetime prevalence of fractures

Men

Age group Ever had a fracture/number of people

21-30 1833/6688 27

$31-40 \quad 3527 / 11519$

$\begin{array}{lll}41-50 & 2128 / 6883 & 31\end{array}$

$51-60 \quad 588 / 1965$

$61-70$

Total
$89 / 300$

$8165 / 27355$

\section{Women}

Ever had a fracture/number

of people

Prevalence (\%)

$1743 / 13003$

$2038 / 13174 \quad 15$

$948 / 5956 \quad 16$

$197 / 943 \quad 21$

$18 / 74 \quad 24$

$4944 / 33150$ included in this report. Other variables included in this analysis are age and gender. Participant age was divided into bands of 10 years (21-30, 31-40, 41-50, 51-60 and 61-70). Age-category analyses presented here excluded seven cohort members aged less than 21 years as they were too few in number to reliably represent that age group. Generally, age groups with very small numbers of people in them were not presented in the age-specific results to prevent distorting age effects.

\section{Analysis}

Only fractures that were reported together with an age at the time of fracture were included in the analysis. Lifetime prevalence of fractures was calculated as the percentage of participants who reported ever having had a fracture. Lifetime incidence of fractures per 100000 person-years was calculated by dividing the number of reported fractures by the sum of the ages of all study participants, and multiplying by 100000 . Fracture incidence was also calculated using a range of recall periods: for example, for a recall period of 10 years, only fractures that were reported to have occurred within the last 10 years were included in the incidence calculation, and the denominator was the number of study participants multiplied by 10 (to account for 10 years of recall). CIs for the incidence rates were calculated by first assuming fracture occurrence to have a Poisson distribution, and finding its related CI. ${ }^{10}$ Differences in recall for leg versus finger/toe fractures were calculated using a $\mathrm{Z}$ test for two proportions: the reported number of fractures over 10 years divided by the expected number of fractures based on 1-year recall multiplied by 10 . All analyses were conducted using SAS software, V.9.2.

\section{RESULTS}

The 60569 TCS participants who responded to the 2009 survey reported a total of 18280 lifetime fractures; age at the time of fractures was included for 18010 (99\%) of these. The overall lifetime prevalence of ever having sustained any fracture was 22\%: there were $47445(78 \%)$ participants who did not report any fracture; $9964(16 \%)$ who reported a fracture at one site; 2146 (4\%) who reported a fracture at two sites and 1014 (2\%) who reported fractures at three or more sites. The distribution of the age-at-fracture as well as the overall study participant age distribution is shown in figure 1. Among participants below the age of 40 , women are in the majority; above 40 years men are in the majority. Although there are more women than men in the study, 65\% (11 645/ $18010)$ of all fractures were reported by men. There was over-reporting of fractures at rounded ages such as 25, 30, 35 and 40 as well as 18 years (figure 1, bottom); a more natural distribution can be achieved by using a 5 -year interval scale.

The prevalence of fractures among men in their 20s and 30s was twice as high as that of women of the same age group (table 1). Having sustained a fracture was more common among women in their 50 s and 60 s than among younger women; among men, there was no marked difference between the age groups.

The fracture incidence per 100000 person-years by fractures site is given in table 2. Finger/toe, arm and ankle fractures were the most common; pelvis and neck fractures the least common. Counting only fractures that occurred in the year prior to the survey resulted in a much higher fracture incidence for most fracture sites. Lifetime fracture incidence includes childhood fractures and relies on accurate recall over a lifetime; fractures over the last year, however, are derived from recent events, and because the youngest participants were 19 years, childhood fractures are not included.

This is further illustrated in figure 2: calculating fracture incidence over a longer recall period resulted in a lower fracture incidence; this effect is seen both in men and women, and across all age groups. The decline in incidence when calculated over longer recall periods is greater for less memorable fractures such as finger/toe, than for fractures associated with greater inconvenience such as leg fractures. On the basis of the number of fractures reported to have occurred in the last year, we can calculate the expected number for a 10 -year period of recall and then compare this to the actual reported number. For finger/toe fractures, the reported number was $55 \%(95 \%$ CI $54 \%$ to $57 \%)$ of the expected, and for leg fractures the corresponding number was $65 \%(95 \%$ 


\begin{tabular}{|c|c|c|c|c|}
\hline \multirow[b]{2}{*}{$\begin{array}{l}\text { Fracture } \\
\text { site }\end{array}$} & \multicolumn{2}{|c|}{ Lifetime fractures } & \multicolumn{2}{|c|}{$\begin{array}{l}\text { Fractures in the last } \\
\text { year }\end{array}$} \\
\hline & $\begin{array}{l}\text { Number of } \\
\text { fractures }\end{array}$ & $\begin{array}{l}\text { Crude } \\
\text { incidence* }^{*}\end{array}$ & $\begin{array}{l}\text { Number of } \\
\text { fractures }\end{array}$ & $\begin{array}{l}\text { Crude } \\
\text { incidencet }\end{array}$ \\
\hline Finger/toe & 3519 & 163 & 268 & 442 \\
\hline Wrist & 1995 & 92 & 98 & 162 \\
\hline Arm & 2967 & 137 & 76 & 125 \\
\hline Collarbone & 1571 & 73 & 62 & 102 \\
\hline Rib & 514 & 24 & 53 & 88 \\
\hline $\begin{array}{l}\text { Face/jaw/ } \\
\text { nose }\end{array}$ & 1006 & 47 & 47 & 78 \\
\hline Neck & 213 & 10 & 21 & 35 \\
\hline Back & 610 & 28 & 73 & 121 \\
\hline Pelvis & 270 & 13 & 19 & 31 \\
\hline Leg & 2007 & 93 & 108 & 178 \\
\hline Ankle & 2051 & 95 & 171 & 282 \\
\hline Other & 1287 & 60 & 134 & 221 \\
\hline Total & 18010 & 834 & 1130 & 1866 \\
\hline \multicolumn{5}{|c|}{$\begin{array}{l}\text { Fracture incidence expressed per } 100000 \text { person-years. The } \\
\text { lifetime fracture crude incidence includes childhood fractures; the } \\
\text { fractures in the last year do not (as the youngest study participant is } \\
19 \text { years). } \\
\text { "Calculated over } 2159580 \text { person-years. } \\
\text { †Calculated over } 60569 \text { person-years. }\end{array}$} \\
\hline
\end{tabular}

CI $62 \%$ to $67 \%$ ). Assuming steady fracture rates over time, the 10-year recall was statistically significantly lower for finger/toe than for leg fractures.

Because the fracture incidence declined when calculated over increasing length of recall, for further analyses only fractures reported to have occurred in the last year were used. Fracture incidence per age group for men and women is shown in figure 3: fractures were more common among young men than among young women; with increasing age, among men the fracture incidence decreased whereas among women fracture incidence increased. Among women above 50 years, fracture incidence was higher than that among men of above 50 years.

\section{DISCUSSION}

This study reports the fracture incidence among Thais aged between 20 and 60 years. Fractures are common, particularly among men under 40 and women above 50 years.

The results of the present study indicate a progressive decline in recall-based fracture incidence over an increasing number of years. Although this pattern appears to be general across age and gender, the magnitude of 'recall inaccuracy' also depends on the severity of the event, with leg fractures being recalled much more accurately than finger/toe fractures. These results, however, should be interpreted with caution as the decline in incidence with increasing recall period could in part be explained by a real increase in fracture

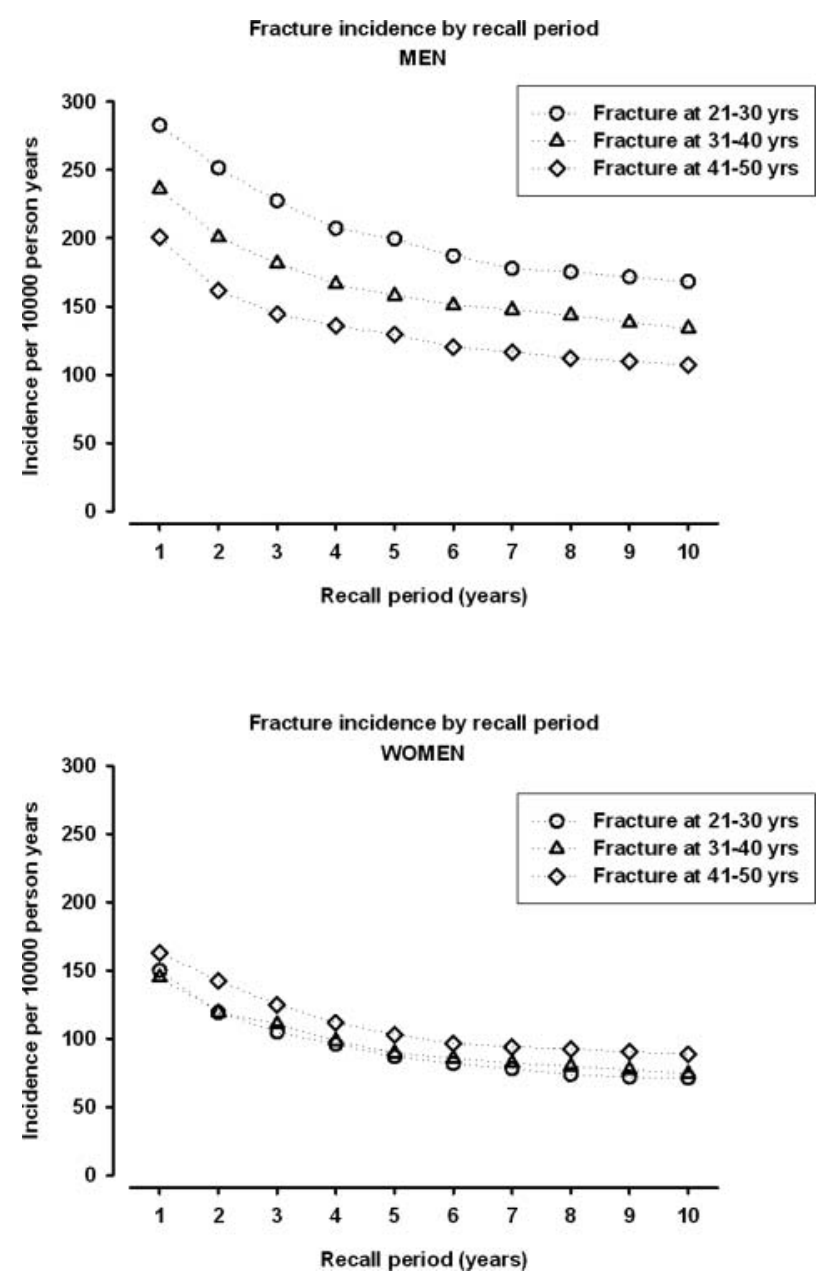

Figure 2 Fracture incidence calculated using a recall period of 1 through to 10 years. Results are shown for fractures that were reported to occur between the ages 20-30, 30-40 and 40-50.

incidence over time. Thailand is transitioning to a modern consumer economy ${ }^{9}$ that could be leading to an increase in fractures due to traffic injury. Baseline frequencies of transport $^{11}$ and other injuries ${ }^{12}$ from the

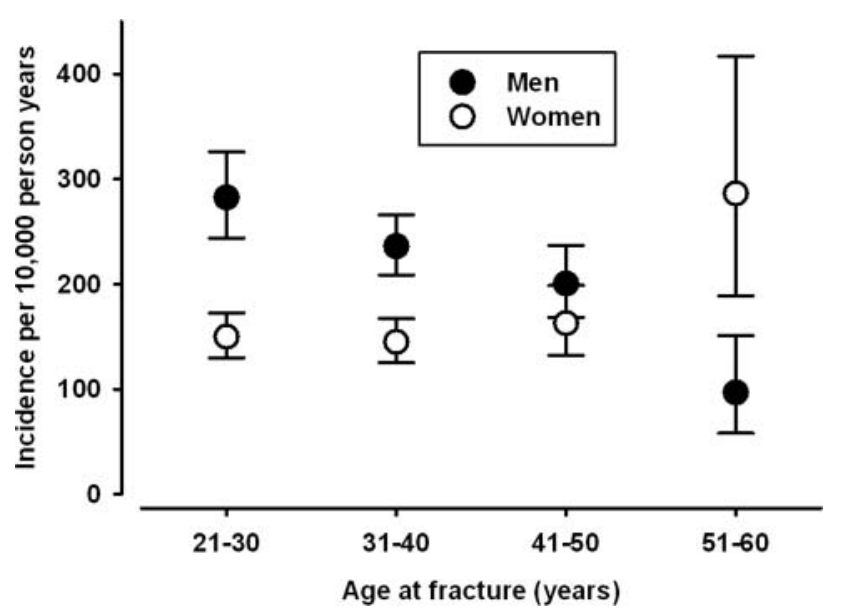

Figure 3 Overall fracture incidence for men and women for a recall period of 1 year. Error bars represent $95 \% \mathrm{Cls}$. 
2005 survey of the TCS have been reported; a change in injury incidence and fracture incidence over time is a topic for further study. Regardless of the underlying change over time, using only the last year of recall to calculate incidence is the most accurate and timely representation of fracture incidence; furthermore, it facilitates comparisons with other studies reporting 12-month recall of fractures.

The overall fracture incidence recorded in the present study is by and large comparable to previous studies that were based on medical records and x-ray reports: some studies reported lower incidence ${ }^{1}{ }^{13-15}$ and others reported similar rates. ${ }^{3} 416$ A recent UK study using a self-report survey reported a fracture incidence twice as high as that presented in this Thai study: ${ }^{5}$ whether this reflects higher fracture rates or better recall in the UK remains to be investigated.

The increase in the incidence of fractures between the ages of 20 and 60 among women and the simultaneous decrease among men seen in this Thai study have been reported previously in studies from the UK, ${ }^{1}{ }^{4} 5$ the USA $^{2}$ and Norway. ${ }^{3}$ Men in their 20 s have previously been reported to have a much higher incidence of fractures than women: this gender difference gradually disappears with age, ${ }^{1514}$ as also seen in the present Thai study.

The pattern of fracture incidence among adult Thais presented here differs from reports from Western studies in two ways. First, the age of onset of a steep increase in fracture rates among women is manifest among the 51-year-old to 60-year-old Thais, but in reports from Western countries ${ }^{41316}$ a steep increase is not apparent until after the age of 65-70 years. This steep increase among older women generally reflects osteoporosis: the present results could therefore indicate earlier onset of osteoporosis among Thai women. This is in agreement with a 2001 study of age-specific osteoporosis prevalence among Thai women which showed that prevalence increased steeply between the ages of 50 and 59 years. ${ }^{17}$ The second discrepancy is the observed decrease in fractures between the ages of 40 and 60 among Thai men. Possibly the decrease in fracture rates after age 20-25 seen among Western men ${ }^{314}$ is not manifest among Thai men until much later because of continued high-risk physical labour; the decrease in fractures after the age of 40 in Thai men could reflect a transitioning out of highrisk work around that age.

This study has several limitations. First, the fracture incidence derived from the TCS 2009 survey is not representative of the Thai population. However, the TCS is a large nation-wide study and the participants represent well the socioeconomic status of the young Thai population. The variations in fracture rates by age and gender observed by internal comparisons in the TCS participants are therefore likely to reflect fracture rate patterns in the general young-adult Thai population. Because fracture incidence is generally described as two-peaked, with greatest incidence in the young and the elderly, ${ }^{1} 35$ the fracture incidence presented in this study is an underestimate of that of the general Thai population, as the young $(<20)$ are not represented and the elderly $(61$ ,$+ n=431$ ) are under-represented. The findings of this study are therefore limited to Thais between the ages of 20 and 60.

Second, the study relies entirely on self-report of recalled fractures. A comparison of self-report with x-ray reports (the gold standard) was not feasible. Instead, we have attempted to quantify self-report (in-) accuracy in two other ways: first, by showing over-reporting of fractures at rounded ages (figure 1). This phenomenon distorts the time distribution rather than the overall number of reported events. The second self-report inaccuracy we have quantified is fall-off over time. Recent events were recalled more accurately than events that occurred longer ago.

\section{CONCLUSIONS}

Self-report surveys provide a feasible alternative to hospital and $\mathrm{x}$-ray records research for establishing fracture incidence; however, when lifetime fractures are reported, using only fractures reported to have occurred in the last year minimises bias due to poor recall. The results of this study indicate that the pattern of fracture incidence among adult TCS participants between 20 and 60 years is similar to that reported for Western countries such as the USA and the UK. Overall, we see fracture in Thailand to be a larger burden for younger men and older women but we need more background information before we can understand better the environmental and personal factors that account for this age and sex pattern of occurrence. Such analyses will be the focus of future reports from the TCS.

\section{Author affiliations}

${ }^{1}$ Monash Injury Research Institute, Monash University, Melbourne, Australia

${ }^{2}$ School of Human Ecology, Sukhothai Thammathirat Open University, Nonthaburi, Thailand

${ }^{3}$ National Centre for Epidemiology and Population Health, Australian National University, Canberra, Australia

Author footnote

'Thai Cohort Study Team: Thailand: Jaruwan Chokhanapitak, Chaiyun Churewong, Suttanit Hounthasarn, Suwanee Khamman, Daoruang Pandee, Suttinan Pangsap, Tippawan Prapamontol, Janya Puengson, Yodyiam Sangrattanakul, Sam-ang Seubsman, Boonchai Somboonsook, Nintita Sripaiboonkij, Pathumvadee Somsamai, Duangkae Vilainerun, Wanee Wimonwattanaphan. Australia: Chris Bain, Emily Banks, Cathy Banwell, Bruce Caldwell, Gordon Carmichael, Tarie Dellora, Jane Dixon, Sharon Friel, David Harley, Matthew Kelly, Tord Kjellstrom, Lynette Lim, Roderick McClure, Anthony McMichael, Tanya Mark, Adrian Sleigh, Lyndall Strazdins, Vasoontara Yiengprugsawan.

Acknowledgements We thank the staff at Sukhothai Thammathirat Open University (STOU) who assisted with student contact and the STOU students who are participating in the cohort study. We also thank Dr Bandit Thinkamrop and his team from Khon Kaen University for guiding us successfully through the complex data processing.

Contributors JG and RM analysed the data and conceptualised and drafted the manuscript. SS and AS designed and instituted the Thai Health-Risk Transition research project and helped the data analysis and 
interpretation for this report. All authors read and approved the final manuscript.

Funding The Thai Cohort Study is funded by the International Collaborative Research Grants Scheme with joint grants from the Wellcome Trust UK (GR071587MA) and the Australian National Health and Medical Research Council (NHMRC; 268055), and as a global health grant from the NHMRC (585426).

Competing interests None.

Ethics approval The approval was obtained from Sukhothai Thammathirat Open University Research and Development Institute (protocol 0522/10) and the Australian National University Human Research Ethics Committee (protocol 2004344).

Provenance and peer review Not commissioned; externally peer reviewed.

Data sharing statement Data are available through a data access agreement which includes guarantees regarding ethical conduct and scientific quality of any proposed analyses and publications. Anyone wanting access should contact Adrian Sleigh or Sam-ang Seubsman (study Principal Investigators).

\section{REFERENCES}

1. Donaldson LJ, Cook A, Thomson RG. Incidence of fractures in a geographically defined population. J Epidemiol Community Health 1990;44:241-5

2. Fife D, Barancik JI. Northeastern Ohio Trauma Study III: incidence of fractures. Ann Emerg Med 1985;14:244-8.

3. Sahlin Y. Occurrence of fractures in a defined population: a 1-year study. Injury 1990;21:158-60.

4. Johansen A, Evans RJ, Stone MD, et al. Fracture incidence in England and Wales: a study based on the population of Cardiff. Injury 1997;28:655-60.

5. Donaldson LJ, Reckless IP, Scholes S, et al. The epidemiology of fractures in England. $J$ Epidemiol Community Health 2008:62:174-80.
6. Pachana NA, Brilleman SL, Dobson AJ. Reporting of life events over time: methodological issues in a longitudinal sample of women. Psychol Assess 2011;23:277-81.

7. Seubsman SA, Lim LL, Banwell C, et al. Socioeconomic status, sex, and obesity in a large national cohort of 15-87-yearold open university students in Thailand. J Epidemiol 2010;20:13-20.

8. Seubsman SA, Yiengprugsawan V, Sleigh A. A large national Thai Cohort Study of the health-risk transition based on Sukhothai Thammathirat Open University students. ASEAN J Open Distance Learning 2010.

9. Sleigh AC, Seubsman SA, Bain C. Cohort profile: The Thai Cohort of 87,134 Open University students. Int J Epidemiol 2008;37:266-72.

10. Altman DGMD, Bryant TN, Gardner MJ Statistics with confidence. 2nd edn. Bristol: BMJ Books, 2000.

11. Stephan K, Kelly M, McClure R, et al. Distribution of transport injury and related risk behaviours in a large national cohort of Thai adults. Accid Anal Prev 2011;43:1062-7.

12. Stephan K, McClure R, Seubsman SA, et al. Review of injuries over a one year period among 87,134 adults studying at an open university in Thailand. Southeast Asian J Trop Med Public Health 2010;41:1220-30.

13. Cooley $\mathrm{H}$, Jones $\mathrm{G}$. A population-based study of fracture incidence in southern Tasmania: lifetime fracture risk and evidence for geographic variations within the same country. Osteoporos Int 2001;12:124-30.

14. Jones G, Cooley HM. Symptomatic fracture incidence in those under 50 years of age in southern Tasmania. J Paediatr Child Health 2002;38:278-83.

15. Sanders KM, Seeman E, Ugoni AM, et al. Age- and gender-specific rate of fractures in Australia: a population-based study. Osteoporos Int 1999;10:240-7.

16. Melton LJ III, Crowson CS, O'Fallon WM. Fracture incidence in Olmsted County, Minnesota: comparison of urban with rural rates and changes in urban rates over time. Osteoporos Int 1999;9:29-37.

17. Limpaphayom KK, Taechakraichana N, Jaisamrarn U, et al. Prevalence of osteopenia and osteoporosis in Thai women. Menopause 2001;8:65-9. 